\title{
Influence of Number of Parity on Bone Mineral Density among Postmenopausal Women
}

\author{
IPALAM $^{\mathrm{a}}$, MAHAQUE ${ }^{\mathrm{b}}$, SB CHOWDHURY ${ }^{\mathrm{c}}$
}

Summary:

Introduction- Osteoporosis is a common disease of postmenopausal women and is responsible for considerable morbidity and mortality. The most important single determining factor is low bone mass. Generally accepted risk factors of osteoporosis in women are low body weight, age, low physical activity and cigarette smoking. The effect of parity is controversial.

Objective-The main objective of the study was to assess the influence of parity on bone mineral density among the postmenopausal women.

Methods-In this study total 75 postmenopausal women aged 51-70 years of with parity 1-13 were studied. Parity was described as the number of births reported by the women. In

\section{Introduction:}

Osteoporosis is one of the long term health problems of postmenopausal women and is responsible for considerable morbidity and mortality. The most important single determining factor is low bone mass. Osteoporosis becomes clinically important only after fracture but treatment of the disorder after the onset of fracture is unsatisfactory ${ }^{1}$ Prevention is, therefore a more effective approach and thus measurement of bone density among postmenopausal women can protect from osteoporosis ${ }^{2}$. Bone densitometry by dual x-ray absorptiometry (DEXA) has been shown to be very reliable and sensitive in diagnosis of osteoporosis and decisions about treatment to prevent fracture. BMD is predominantly regulated by genetic. The rest of factors/ variance is influenced by environmental factors, such

a. Dr. Irin Parveen Alam, Assistant Prof., Obstetrics \& Gynae, Sir Salimullah Medical College and Mitford Hospital.

b. Dr. Mohd Azharul Haque, Assistant Prof (Medicine), Sir Salimullah Medical College, Dhaka.

c. Prof. Saleha Begum Chowdhury, Chairman and Head of Department of Obstetrics \& Gynaecology. Bangabandhu Sheikh Mujib Medical Uni versity. Dhaka.

Address of Correspondence: Dr. Irin Parveen Alam, Assistant Prof Obstetrics \& Gynae, Sir Salimullah Medical College and Mitford Hospital., Mobile: 88-01715348398, E-mail: dririn. alam@yahoo.com

Received: 15 December, 2013 Accepted: 10 December, 2014 this study $T$ score of BMD of different bony sites lumber vertebrae and femur were analyzed. BMD were measured in the Institute of Nuclear Medicine at BSMMU. Correlations between BMD values with parity were detected.

Results- The mean age of the patients was 60 years with a standard deviation of \pm 9.32 years. All patients were within 51 to 70 years age range. A significant negative correlation was found in present study between parity and the $T$ score measurement results obtained from L2, L3, L4, L2-4, Femur neck, Trochantor and Ward's triangle. This shows mean Tscore of $B M D$ were more negative as number of parity increases.

Key words: BMD. Postmenopausal women, Parity.

(J Banagladesh Coll Phys Surg 2015; 33: 75-78)

as a pregnancy or a period of lactation. ${ }^{3 \text {. Findings }}$ about the relation between parity and BMD are controversial ${ }^{4}$. So far in our country no study has been conducted to see influence of parity on BMD among the postmenopausal women. A better understanding of this relation may provide new opportunity for early intervention.

Objective-The main objective of the study was to assess the influence of parity on bone mineral density among the postmenopausal women.

Methods- This cross sectional observational study was carried out from the Jan 2006-Dec 2007 for a period of two years. This study was carried out in the Department of Obstetrics and Gynaecology Bangabandhu Sheikh Mujib Medical University (BSMMU) in collaboration with the Institute of Nuclear Medicine and Ultrasound, BSMMU campus, Dhaka. Ethical clearance was taken from the ethical committee of Department of Obstetrics and Gynaecology of BSMMU. Sampling technique was purposive consecutive sampling. The study population consisted of patients who had natural menopause from the age 51-70 years, attending the outpatient department of Department of Obstetrics and Gynaecology BSMMU Hospital and interested to perform BMD test and giving consent to participate in the study. Patients having menopause before the age of 50 years or patients having surgical menopause, history of fracture, chronic medical disorders affecting the 
bone mineral density such as chronic renal disorder, hypo or hyperthyroidism and medications known to affect the bone such as anticoagulant and anticonvulsant were excluded from the study. Cases were collected from outpatient department of Obstetrics and Gynaecology department of BSMMU. After describing the purpose and procedure of the study informed written consent was taken from every patient. All the patients answered the same specially designed questionnaire. Height and weight were measured with the participant wearing light clothing and no shoes. Body mass index (BMI) was used as an estimate of obesity. Bone mass density was measured at the Institute of Nuclear Medicine, Bangabandhu Sheikh Mujib Medical University (BSMMU), Dhaka by dual energy xray absorptiometry (DEXA), at the lumber spine and femoral neck by technician of BMD laboratory. The analysis is a computer-automated analysis and the measured BMD automatically displayed. Based on the definition of World Health Organization (WHO), T score of Bone Mineral Density values of different bony site such as lumber vertebra 2-4 and femoral neck were considered for analysis. Age of patients was divided into 4 groups, at 5 years interval such as 51-55years, 56-60years, 61-65 years and 66-70 years.

Data analysis was done by using SPSS by applying appropriate statistical formula such as unpaired t test, Chi squired test. Spearman's correlation and Pearson's correlation test were employed to measure the relation between the variable studied. The level of statistical significance was set at a p value of $<0.05$.

\section{Results:}

The present study was carried out in BSMMU from January 2006-December 2007. The study included 75 postmenopausal women ages of patients were within 51-70 years.

Table-I

Age distribution of the study subjects.

\begin{tabular}{lccc} 
Age (year) & Frequency & Percent & Mean \pm SD (range) \\
\hline $51-55$ & 18 & 24.0 & \\
$56-60$ & 27 & 36.0 & \\
$61-65$ & 18 & 24.0 & $60.0 \pm 9.32(51-70)$ \\
$66-70$ & 12 & 16.0 & \\
\hline Total & 75 & 100.0 & \\
\hline
\end{tabular}

Out of all patients maximum 27 (36.0\%) were within 56 to 60 years age range followed by 18 (24.0\%) within 51 to 55 years $18(24.0 \%)$ within $61-65$ years and $12(16.0 \%)$ within
66 to 70 years age range. Mean age of the patients was 60 years with a standard deviation of \pm 9 .32 years. All patients were within 51 to 70 years age range. (Table I)

Table-II

Demographic related characteristics of the patients

Demography related factors

Mean \pm SD (range)

Age of menarche (year)

$13.09 \pm 0.89(10-15)$

Age of menopause (year)

$53.68 \pm 3.93(50-57)$

Duration of menopause (year)

$11.41 \pm 7.95(1-20)$

Age of marriage (year)

$16.65 \pm 2.50(11-26)$

Age of first pregnancy (year)

$19.28 \pm 2.67(15-28)$

Para

$6.07 \pm 3.23(1-13)$

Total duration of breast

$133.35 \pm 69.89(2-170)$

feeding (month)

Among the 75 patients, age of menarche were from 10 15 years, age of menopause ranges from 51-57 years, duration of menopause ranges from 1-20 years .Age of marriage were from 11-26 years, most of the patients age of first pregnancy was at the age of 15-28years. Among the patients parity varied from 1 to 13 . As parity varies duration of breastfeeding varied according to number of children and it varied from 2-170 months.

\section{Table-III}

\begin{tabular}{|c|c|c|}
\hline T score vs. parity & rvalue $^{\S}$ & p value \\
\hline $\mathrm{L}_{2}$ & $-0.413(* *)$ & 0.001 \\
\hline $\mathrm{L}_{3}$ & $-0.402(* *)$ & 0.001 \\
\hline $\mathrm{L}_{4}$ & $-0.437(* *)$ & 0.001 \\
\hline $\mathrm{L}_{2-4}$ & $-0.436(* *)$ & 0.001 \\
\hline Femur neck & $-0.321(* *)$ & 0.005 \\
\hline Trochantor & $-0.308(* *)$ & 0.007 \\
\hline Ward's triangle & $-0.291\left(^{*}\right)$ & 0.011 \\
\hline
\end{tabular}

A significant negative correlation was found between parity and the $\mathrm{T}$ score measurement results obtained from L2, L3, L4, L2-4, Femur neck, Trochanter and Ward's triangle $(\mathrm{p}<0.001$ in all areas, $\mathrm{r}=-0.413, \mathrm{r}=-0.402, \mathrm{r}=$ $0.437, r=-0.436, r=-0.321, r=-0.308$ and $r=-0.291$ respectively). Table-III 


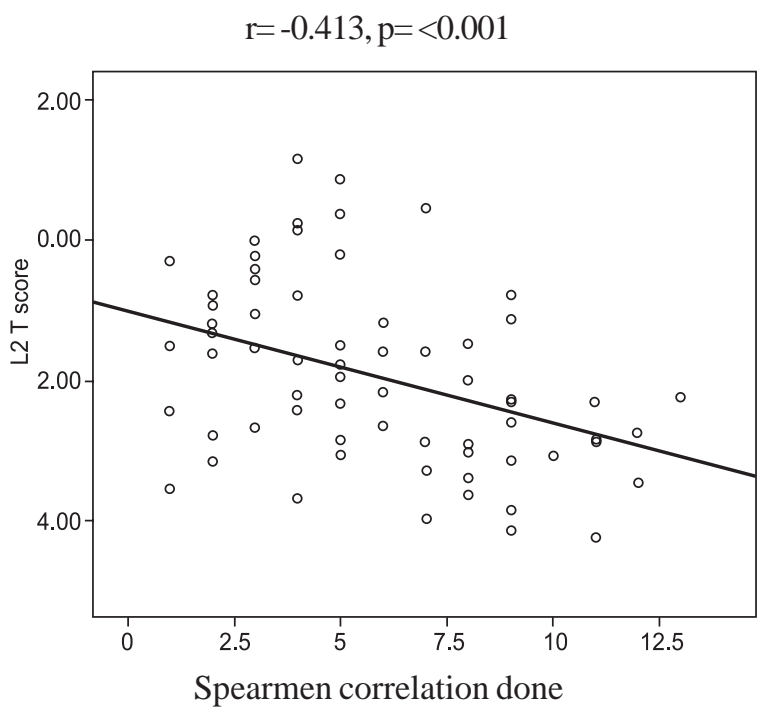

Fig.-1: Scatter plot of correlation of T score (L2) and number of parity

Figure-1 shows a significant negative correlation was found between parity and T score of BMD of lumber 2 vertebra. The value of Spearman correlation coefficient was -0.413 which indicates negative correlation between this two variables and it is highly significant, p value $<0.001$.

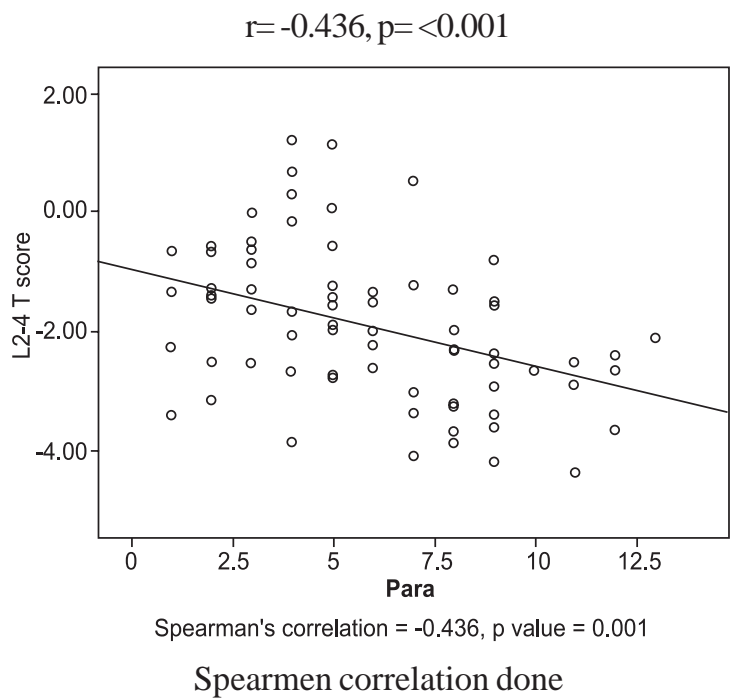

Fig.-2: Scatter plot of correlation of T score $\left(L_{2-4}\right)$ and number of parity

Fig 2 shows a significant negative correlation between parity and T score of BMD of lumber 2-4 vertebra. The value of Spearman correlation coefficient was -0.436 which indicates negative correlation between parity and lumber 2-4 vertebra and it is highly significant, p value<
0.001 which indicates that with increasing parity $\mathrm{T}$ score of BMD of lumber 2-4 vertebrae decreases.

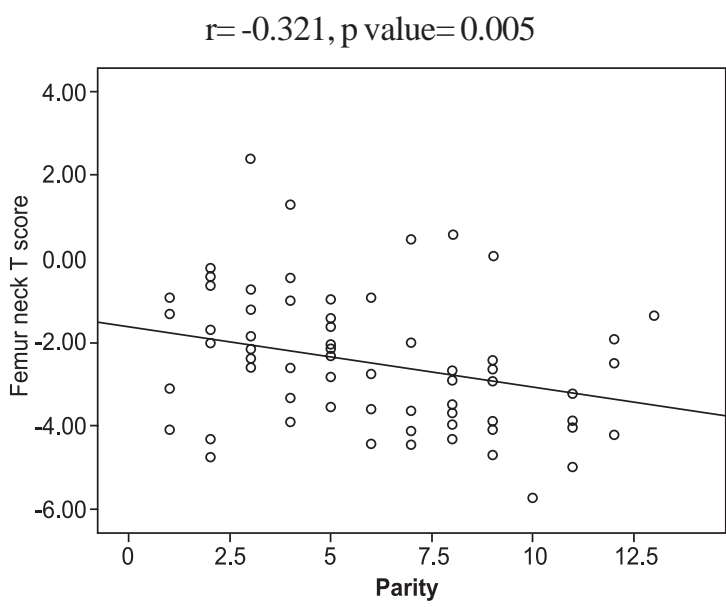

Spearman's correlation $=-0.321, p$ value $=0.005$

Spearmen correlation done

Fig.-3: Scatter plot of correlation of T score (Femur neck) and number of parity

Fig 3 shows a significant negative correlation between parity and T score of BMD of femur neck. The value of Spearman correlation coefficient was -0.321 which indicates negative correlation between parity and femur neck and it is significant, $p$ value $<0.01$. This indicates that with increasing parity $\mathrm{T}$ score of BMD of femur neck decreases.

\section{Discussion:}

This cross sectional study was carried out in Bangladeshi postmenopausal women. The main aim of the study was to assess the influence of parity on bone mineral density among the postmenopausal women. For these purpose total 75 postmenopausal women aged 51-70 years of with parity 1-13 were studied. Parity was described as the number of births reported by the women.

World Health Organization (WHO) has published a guideline for the diagnosis of osteopenia and osteoporosis, which is related to an individual's BMD to the peak bone density of young adult (T-score). Osteoporosis is defined as a BMD of more than 2.5 SD below that of young adults (T-score -2.5 or more) where as a BMD between one and 2.5SD below that of young adults (T-score -1 to -2.5 ) is considered osteopenia. ${ }^{5}$.

In this study $\mathrm{T}$ score of BMD of different bony sites lumber vertebrae and femur were analyzed. In this study the mean age of the patients was 60 years with a standard deviation of \pm 9.32 years.

A significant negative correlation was found in present study between parity and the $\mathrm{T}$ score measurement 
results obtained from L2, L3, L4, L2-4, Femur neck, Trochanter and Ward's triangle. In a study conducted by Ozdemir et al. significant negative correlations were found between the number of pregnancies and BMD values for the spine and femur (neck, trochanter) which is resembled with present study, ${ }^{6,7}$ In the present study mean $\mathrm{T}$ score of BMD of lumber 2,3,4,2-4 and femur neck, trochantor wards triangle were observed more negative as the number of parity increase. A study showed that BMD in healthy Saudi females was lower than that of their USA counterparts. That study also found that BMD value decrease as the number of parity increases. They contributed that to increase number of pregnancies, longer duration of lactation with prevalent vitamin D deficiency ${ }^{8}$. Hreshchyshyn et al. reported that the BMD of the femoral neck declined with an increasing number of live births, this finding is similar with present study but they found no change in lumber spine. ${ }^{9}$ Fox et al. had observed a $1.4 \%$ increase in distal radius bone density with every pregnancy. In a study carried out by Hoffman et al. (1993), where the relation between parity and risk of fracture was investigated, among women who had three or more children, the risk of fracture were found $35-40 \%$ lower than that of nulliparous women. The study stated that BMD increases in subsequent pregnancies. These findings do not correlate with present study. ${ }^{10}$

Gur et alfound a significant negative correlation between the number of pregnancies and BMD of the spine, trochantor, and Wards triangle. But in the present study a significant negative correlation was found between number of pregnancies and spine, trochantor, Wards triangle and also femur neck. ${ }^{4}$ These findings correlate with present study.

Carranza et al. found no significant correlation between the numbers of pergnancy $\mathrm{T}$ scores at lumber spine and femur. But in this study a significant negative correlation was found between the numbers of pregnancy and $\mathrm{T}$ scores at lumber spine and femur. ${ }^{11}$

The present study revealed a negative and correlation between increasing parity and decreasing BMD at lumber vertebrae and hip. The BMD values found decreased as the number of parity increased.

This study was conducted in one tertiary hospital with a small sample size so it reflects a small population. Hormonal factors that influence osteoporosis were not measured in this study. History of oral contraceptive intake should be evaluated for further study. Further prospective studies with a large sample should be carried out for evaluation of an association between parity and bone mineral density.

Conclusion- The results of the study conclude that among the postmenopausal women ,T score of bone mineral density decreases as the number of parity increases.

Acknowledgement: The author is highly grateful to Institute of nuclear medicine BSMMU and Department of Obstetrics and Gynaecology BSMMU and also Prof. Mozammel Haque Department of Biochemistry for his encouragement.

\section{References:}

1. Karlsson M, Henrik GA, Karlsson C . Maternity and bone mineral density. Acta Orthopaedica.2005; 76 (1): 2-13.

2. Panay N . Menopause and the postmenopausal women. In: Edmons DK (eds), Dewhurst's text book of Obstetrics \& Gynaecology, $7^{\text {th }}$ ed, Blackwell publishing, Oxford; 2007. 479-492.

3. Chan GM, Slater P, Ronald N, Roberts CC, Thomas MR, Folland D, Jackson R . Bone mineral status of lactating mothers of different ages. Am J Obstet Gynecol.1982; 144:438-41

4. Gur A, Nas K, Cevik R, Sarac AJ, Ataoglu S, Karakoc M. The influence of duration of breastfeeding on bone mass in postmenopausal women of different age groups. Int $\mathrm{J}$ Clin Pract.2003; 57(2):82-6.

5. World Health Organization . Assessment of fracture risk and its application to screening for postmenopausal osteoporosis. Technical report series. Geneva: WHO1994; 843

6. Kojima N, Douchi T, Kosha S, Nagata Y . Cross-sectional study of the effects of parturition and lactation on bone mineral density later in life. Maturitas 2002;41:203-209

7. Ozdemir F, Demirbag D, Meliha R. Reproductive factors Affecting the Bone Mineral Density in Postmenopausal Women. J Exp Med.2005; 205:277-285

8. Ghannam NN, Hammami MM, Bakheet SM, Khan BA . Bone mineral density of the spine and femur in healthy Saudi females: relation to vitamin D status, pregnancy and lactation. Calcif Tissue Int.1999; 65: 23-28.

9. Hreshchyshyn MM, Hopkins A, Zylsta S, Anbar M . Associations of parity, breastfeeding and birth control pills with lumber spine and femoral neck bone densities. Am J Obstet Gynecol.1988; 159:318-322.

10. Fox KM, Magaziner J, Sherwin R, Scott JC, Plato CC, Nevitt M, Cummings S. Reproductive correlates of bone mass in elderly women: The Study of Osteoporotic Fractures Research Group. 1993 J Bone Mi Res ner 8: 901-908.

11. Carranza-LS \& Mera JP. Influence of number of pregnancies and total breast-feeding time on bone mineral density 2002. Int J Fertil Women Med 147:169-171. 